\title{
A REPRESENTATION THEOREM FOR BOUNDED CONVEX SETS
}

\author{
R. R. PHELPS
}

1. Introduction. It is well known that any closed convex subset of a normed linear space can be represented as the intersection of all those closed half-spaces which contain it. It is also well known (and easy to prove) that if the closed convex set is bounded, and if the space under consideration is Euclidean $n$-space, then the set can be represented as the intersection of cells, i.e., the following is true in the case when $E$ is Euclidean $n$-space:

If $C$ is a bounded closed convex subset of the normed linear space $E$, and if $x \in E \sim C$, then there exist $y \in E$ and $r>0$ such that the cell $N_{r} y=\{z:\|z-y\| \leqq r\}$ contains $C$ but not $x$.

In the present paper we consider those spaces $E$ for which the above statement holds for all such $C$; such a space is said to have the property (g): Every bounded closed convex set $C$ can be represented as the intersection of cells.

Our study was motivated in part by a paper of S. Mazur [5], in which property (g) was shown to hold for all reflexive Banach spaces having a strongly differentiable norm, and in which the following criterion for weak sequential convergence was proved:

Suppose $E$ has property (g). Then a sequence $\left\{x_{n}\right\}$ in $E$ converges weakly to the point $x$ if and only if (i) the sequence $\left\{x_{n}\right\}$ is bounded and (ii) every cell containing infinitely many $x_{n}$ also contains $x$.

In what follows we obtain a general sufficient condition for property (g), as well as some necessary conditions, these conditions all being given in the form of density criteria for certain subsets of $U^{*}$, the unit cell of the conjugate space $E^{*}$. For finite dimensional $E$, these conditions are equivalent, and we obtain the following interesting result:

$A$ finite dimensional space $E$ has property (g) if and only if the set of extreme points of $U^{*}$ is dense in the boundary of $U^{*}$.

An example is given which shows that in infinite dimensional spaces, the above condition (while necessary) does not imply property (g). The same example provides an answer to a question of Mazur [5], by showing the existence of a Banach space in which the norm is

Presented to the Society, January 29, 1960; received by the editors November 27, 1959 and, in revised form, February 11, 1960. 
weakly differentiable everywhere (except at the origin) but strongly differentiable nowhere.

Before proceeding, we list some definitions used throughout the paper. Denote the set of real numbers by $R$. Let $S$ and $S^{*}$ denote the unit spheres of $E$ and $E^{*}$ respectively, i.e., those elements of norm 1, and let $U$ and $U^{*}$ denote the unit cells of $E$ and $E^{*}$, i.e., those elements of norm at most 1 .

2. A sufficient condition for property (g). If $C$ is a bounded, closed, convex subset of a normed linear space $E$ such that $\phi \notin C$, let $C^{\prime}$ be the set of all linear functionals $f$ in $E^{*}$ such that

$$
\inf f(C)(=\inf \{f(x): x \in C\})
$$

is positive. It follows from the basic separation theorem [1] that $C^{\prime}$ is nonempty. A subset $K$ of a linear space is called a convex cone if $K$ is convex and if $x \in K$ and $\lambda>0$ imply $\lambda x \in K$.

Lemma 2.1. If $C$ is a bounded, closed convex subset of a normed linear space $E$, then $C^{\prime}$ is an open convex cone in $E^{*}$.

Proof. It is easily verified that $C^{\prime}$ is a convex cone. To see that $C^{\prime}$ is open, suppose that $f \in C^{\prime}$, let $\alpha=\inf f(C)>0$, and choose $M>0$ such that $C \subset N_{M} \phi$. If $\|f-g\|<\alpha / 2 M$, then for each $x \in C$ we have $f(x)$ $-g(x) \leqq\|f-g\|\|x\|<\alpha / 2$, so that $g(x) \geqq f(x)-\alpha / 2 \geqq \alpha / 2>0$. Hence inf $g(C)>0$, which shows that $g \in C^{\prime}$ and therefore $C^{\prime}$ is open.

We say that the norm in $E$ is strongly differentiable at the point $x \neq \phi$ if there exists an $f$ in $S^{*}$ such that $\lim _{y \rightarrow 0} \delta_{x}(y) /\|y\|=0$, where $\delta_{x}(y)=\|x+y\|-\|x\|-f(y)$. Let $\operatorname{str} S^{*}$ be the set of all $f$ in $S^{*}$ which attain their norm at a point of strong differentiability, i.e., for which there exists an $x$ in $S$ such that the norm in $E$ is strongly differentiable at $x$ and $f(x)=1$. The following theorem, while more general than Mazur's theorem [5], is proved in much the same way.

Theorem 2.2. A normed linear space $E$ has property (g) if $\operatorname{str} S^{*}$ is dense in $S^{*}$.

Proof. Suppose that $C$ is a bounded, closed convex subset of $E$ and that $y \in E \sim C$. We may assume, without loss of generality, that $y=\phi$. Since $\operatorname{str} S^{*}$ is dense in $S^{*}$ and (by Lemma 2.1) $C^{\prime} \cap S^{*}$ is open in $S^{*}$, there exists an $f$ in $\operatorname{str} S^{*}$ and an $x$ in $S$ such that inf $f(C)>0$, $f(x)=1$, and the norm in $E$ is strongly differentiable at $x$. Choose $\epsilon>0$ such that inf $f(C)>2 \epsilon>0$ and let $z=\epsilon x$. For each $r>1$ let $N_{r}$ be the cell of radius $\|(r-1) z\|=(r-1) \epsilon$ which is centered at $r z$. The origin is not in $N_{r}$ for any $r>1$, so it will suffice to show that $C \subset N_{r}$ for some $r>1$. Suppose not; then there exist sequences $\left\{r_{n}\right\}$ and $\left\{x_{n}\right\}$ 
such that $r_{n}>1, r_{n} \rightarrow \infty,\left\|x_{n}-r_{n} z\right\|>\left(r_{n}-1\right) \epsilon$ and $x_{n} \in C$ for each $n$. The sequence $\left\{x_{n}\right\}$ is bounded because $C$ is bounded, so we have $y_{n}=-x_{n} / \epsilon r_{n} \rightarrow \phi$ as $n \rightarrow \infty$. Hence, by strong differentiability of the norm at $x$, we must have $\delta_{x}\left(y_{n}\right) /\left\|y_{n}\right\| \rightarrow 0$ as $n \rightarrow \infty$. But $\epsilon r_{n} \delta_{x}\left(y_{n}\right)$ $=\left\|r_{n} z-x_{n}\right\|-\epsilon r_{n}+f\left(x_{n}\right)>\left(r_{n}-1\right) \epsilon-\epsilon r_{n}+2 \epsilon=\epsilon$. Hence

$$
\delta_{x}\left(y_{n}\right) /\left\|y_{n}\right\|=\epsilon r_{n} \delta_{x}\left(y_{n}\right) /\left\|x_{n}\right\|>\epsilon /\left\|x_{n}\right\|,
$$

a contradiction, since the $x_{n}$ are bounded.

If $E$ is a normed linear space let $P(E)$ be the set of all those linear functionals $f$ in $E^{*}$ which attain their supremum on $S$, i.e. those $f$ for which there exists $x$ in $S$ such that $f(x)=\|f\|$. We call $E$ subreflexive if $P(E)$ is norm-dense in $E^{*}$, or equivalently, if $P(E) \cap S^{*}$ is dense in $S^{*}$. Note that, always, $\operatorname{str} S^{*} \subset P(E) \cap S^{*}$, so it follows immediately from our hypothesis in Theorem 2.2 that $E$ is subreflexive. In Theorem 4.3 (i) it will be shown that subreflexivity is a necessary condition of property (g). (There exist non-subreflexive normed linear spaces [6; see also 7] but, to the best of our knowledge, the question "Is every Banach space subreflexive?" remains open.) ${ }^{1}$

3. An elementary lemma. In this section we prove a lemma which describes a basic connection between pairs of linear functionals and the hyperplanes determined by them. Roughly speaking, the lemma asserts the intuitively obvious fact that if two functionals $f$ and $g$ of norm one have their hyperplanes $f^{-1}(0)$ and $g^{-1}(0)$ sufficiently close together, then one of $\|f-g\|,\|f+g\|$ must be small.

Lemma 3.1. Suppose that $E$ is a normed linear space and that $\epsilon>0$. If $f, g \in S^{*}$ are such that $f^{-1}(0) \cap U \subset g^{-1}[-\epsilon / 2, \epsilon / 2]$, then either $\|f-g\|$ $\leqq \epsilon$ or $\|f+g\| \leqq \epsilon^{2}$

Proof. By the Hahn-Banach theorem we can choose $h$ in $E^{*}$ such that $h=g$ on $f^{-1}(0)$ and $\|h\|=\sup \left|g\left(U \cap f^{-1}(0)\right)\right|$. Then, by hypothesis, $\|h\| \leqq \epsilon / 2$. Furthermore, since $g-h$ vanishes on $f^{-1}(0)$ there exists $\alpha$ in $R$ such that $g-h=\alpha f$. Hence $\|g-\alpha f\|=\|h\| \leqq \epsilon / 2$. Assuming $\alpha \geqq 0$, we will show that $\|f-g\| \leqq \epsilon$. (Otherwise, the same proof applied to $(-\alpha) f$ would show that $\|f+g\| \leqq \epsilon$.) If $\alpha \geqq 1$, then $\alpha^{-1} \leqq 1$ and $\|g-f\|=\left\|\left(1-\alpha^{-1}\right) g+\alpha^{-1}(g-\alpha f)\right\| \leqq 1-\alpha^{-1}+\alpha^{-1}\|g-\alpha f\|$. Also, $\alpha=\|\alpha f\| \leqq\|g\|+\|g-\alpha f\|$ so $1-\alpha^{-1} \leqq(1+\|g-\alpha f\|)^{-1}\|g-\alpha f\| \leqq\|g-\alpha f\|$. Hence $\|g-f\| \leqq 2\|g-\alpha f\| \leqq \epsilon$. If $0 \leqq \alpha<1$, then $\|g-f\| \leqq\|g-\alpha f\|$ $+\|(1-\alpha) f\|=\|g-\alpha f\|+1-\alpha=\|g-\alpha f\|+\|g\|-\|\alpha f\| \leqq 2\|g-\alpha f\| \leqq \epsilon$, which completes the proof.

${ }^{1}$ Note added in proof: See Errett Bishop and R. R. Phelps, $A$ proof that every Banach space is subreflexive, Bull. Amer. Math. Soc., to appear.

2 It should be noted that this lemma provides a short proof of Theorem 1.2 of [6]. 
4. Some consequences of property (g). If $x$ is in $S$, let $F_{x}$ be the face of $S^{*}$ determined by $x$, i.e., $F_{x}=\{f:\|f\|=1=f(x)\}$. Each such face is closed, convex and (by the Hahn-Banach theorem) nonempty. The next lemma is basic to the rest of the paper.

LEMma 4.1. Suppose that the normed linear space $E$ has property (g). If $f \in S^{*}$ and $0<\epsilon<1$ there exist $\delta(\epsilon)>0$ and $x \in S$ such that $y \in S \cap N_{\delta} x$ implies $F_{y} \subset N_{f}$.

Proof. If $f \in S^{*}$ and $0<\epsilon<1$ let $D=U \cap f^{-1}(0)$ and pick $u \in S$ such that $f(u)>\epsilon$. Let $u^{\prime}=(\epsilon / 2) u$. Since $D$ is bounded, closed and convex, and $u^{\prime} \notin D$, property (g) implies the existence of $r>0$ and $z \in E$ such that $u^{\prime} \notin N_{r} z \supset D$. Let $w$ be the intersection of the segment $\left[u^{\prime}, z\right]$ with the boundary of $N_{r} z$; then if $C$ denotes the convex hull of $u^{\prime}$ and $N_{r} z$ we can conclude that $w \in \operatorname{int} C$, the interior of $C$. Hence there exists $\alpha>0$ such that $\|v-w\| \leqq \alpha$ implies $v \in \operatorname{int} C$. Note that for any such $v$ there exists $v^{\prime} \in \operatorname{int} N_{r} z$ and $\left.\lambda \in\right] 0,1$ [ such that $v=\lambda u^{\prime}+(1-\lambda) v^{\prime}$.

Now let $x=r^{-1}(w-z)$ and let $\delta=\alpha r^{-1}$. If $y \in S \cap N_{\delta} x$ and $g \in F_{y}$ then $g(y)=\|g\|=1=\sup g(U)$. Letting $v=r y+z$ we have $g(v)$ $=r \sup g(U)+g(z)=\sup g\left(N_{r} z\right)$. Now $\|v-w\|=\|v-(r x+z)\|=r\|x-y\|$ $\leqq r \delta=\alpha$, so there exist $v^{\prime}$ and $\lambda$ as above. Since $v^{\prime} \in$ int $N_{r} z$, we have $g\left(v^{\prime}\right)<g(v)$, while $\phi \in D \subset N_{r} z$ implies $g(v) \geqq 0$. Hence $g(v)=\lambda g\left(u^{\prime}\right)$ $+(1-\lambda) g\left(v^{\prime}\right)<\lambda g\left(u^{\prime}\right)+(1-\lambda) g(v)$ or $0 \leqq g(v)<g\left(u^{\prime}\right)$. Since $g(u) \leqq\|g\|$ - $\|u\|=1, g\left(u^{\prime}\right)=(\epsilon / 2) g(u) \leqq \epsilon / 2$ and therefore $g(v)<\epsilon / 2$. Thus, for any $w^{\prime} \in D \subset N_{r} z, g\left(w^{\prime}\right) \leqq g(v)<\epsilon / 2$ and, by the symmetry of $D$ about $\phi, g\left(w^{\prime}\right)>-\epsilon / 2$. Hence $D=f^{-1}(0) \cap U \subset g^{-1}[-\epsilon / 2, \epsilon / 2]$ and therefore, by Lemma 3.1, either $\|f-g\| \leqq \epsilon$ or $\|f+g\| \leqq \epsilon$. But $g(u)>(2 / \epsilon) g(v)$ $\geqq 0$ and $f(u)>\epsilon$, so $\epsilon<(f+g)(u) \leqq\|f+g\|$, which shows that $\|f-g\|$ $\leqq \epsilon$. Since this is true for any $g \in F_{y}$, we have $F_{y} \subset N_{\epsilon} f$.

If $K$ is a convex subset of a linear space we say that a subset $A$ of $K$ is an extremal subset of $K$ if $A$ is nonempty and both $A$ and $K \sim A$ are convex. An extremal subset of $K$ which consists of a single point is called an extreme point of $K$. It is easy to verify that if $x \in S$ then $F_{x}$ is an extremal subset of $U^{*}$, the unit cell of $E^{*}$. Furthermore, the set $\{f: f(x)=1\}$ is weak ${ }^{*}$-closed in $E^{*}$ and hence, since $U^{*}$ is weak ${ }^{*}$ compact, $F_{x}=U^{*} \cap\{f: f(x)=1\}$ is weak*-compact. It follows from the Krein-Milman theorem [1] that $F_{x}$ has at least one extreme point, and (using the fact that $F_{x}$ is extremal in $U^{*}$ ) this point is also an extreme point of $U^{*}$. If $B$ is a convex set, let ext $B$ be the set of all extreme points of $B$.

Let $\varphi$ be a "selection" for the mapping $x \rightarrow F_{x}$ such that, for each $x \in S, \varphi(x)$ is an extreme point of $F_{x}$. If $A$ is any subset of $S$, then the cardinality of $\varphi(A)=\{\varphi(x): x \in A\}$ is no greater than that of $A$. We have the following immediate (and useful) corollary to Lemma 4.1. 
COROLlaRY 4.2. If $E$ is a normed space having property (g), and if $A$ is dense in $S$, then $\varphi(A)$ is dense in $S^{*}$.

We say that $x \in S$ is a smooth point of $S$ if $F_{x}$ consists of exactly one point. Denote the set of smooth points of $S$ by sm $S$. A point $y$ of a convex set $K \subset E$ is called an exposed point of $K$ if there exists a hyperplane $H$ supporting $K$ at $y$ such that $H \cap K=\{y\}$. Equivalently, $y \in \exp K$ (the set of all exposed points of $K$ ) if and only if there exists a linear functional $f \in E^{*}$ such that $\sup f(K)=f(y)$ and $f(z)<f(y)$ for $z \in K \sim\{y\}$. Note that, always, $\exp K \subset \operatorname{ext} K$. If $x \in \operatorname{sm} S$ and $f$ is the unique point of $F_{x}$, consider $x$ as a continuous linear functional on $E^{*}$ (by letting $x(g)=g(x)$ for each $g \in E^{*}$ ); then if $g \in U^{*} \sim\{f\}, x(g)<1=x(f)$, so $f \in \exp U^{*}$. Thus, the following inclusions always hold: $\varphi(\operatorname{sm} S) \subset \exp U^{*} C \operatorname{ext} U^{*}$.

THEOREM 4.3. Suppose that $E$ is a normed linear space having property $(g)$; then the following implications are valid:

(i) The set $\varphi(S)\left(\subset P(E) \cap\right.$ ext $\left.U^{*}\right)$ is dense in $S^{*}$; hence $E$ is subreflexive and ext $U^{*}$ is dense in $S^{*}$.

(ii) If $\operatorname{sm} S$ is dense in $S$, then $\varphi(\operatorname{sm} S)\left(C \exp U^{*}\right)$ is dense in $S^{*}$.

(iii) If $E$ is separable, then $E^{*}$ is separable.

(iv) If $E$ is separable and complete, then $\exp U^{*}$ is dense in $S^{*}$.

Proof. All the statements, with the exception of (iv), follow trivially from Corollary 4.2 and the preceding remarks and definitions. To prove (iv), we use the theorem of Mazur [4] which states that, for separable Banach spaces, $\operatorname{sm} S$ is dense in $S$; (ii) now yields (iv).

THEOREM 4.4. If $E$ is a finite dimensional normed linear space the following assertions are equivalent:

(i) The space $E$ has property (g).

(ii) The set $\exp U^{*}$ is dense in $S^{*}$.

(iii) The set ext $U^{*}$ is dense in $S^{*}$.

Proof. That (i) implies (iii) follows from Theorem 4.3 (i). By a theorem of Klee [3, Theorem 2.3], exp $U^{*}$ is dense in ext $U^{*}$, so (iii) implies (ii). To show that (ii) implies (i), we first show that $\exp U^{*}=\operatorname{str} S^{*}$ in any finite dimensional space, then we apply Theorem 2.2. Mazur has pointed out $[5$, p. 130] that, in a finite dimensional space $E$, a point $x$ is in $\mathrm{sm} S$ if and only if the norm in $E$ is strongly differentiable at $x$. Thus, if $f \in \operatorname{str} S^{*}, f$ attains its supremum at a smooth point of $S$ and therefore, using the remarks preceding Theorem 4.3, $f \in \exp U^{*}$. On the other hand, if $f \in \exp U^{*}$, there exists $X$ in $E^{* *}$ such that $X(f)=1=\|X\|$ and $X(g)<1$ for $g \in U^{*}$ 
$\sim\{f\}$. From the reflexivity of finite dimensional spaces it follows that there exists an $x \in S$ such that $g(x)=X(g)$ for each $g \in E^{*}$. From the above conditions on $X$ we conclude that $F_{x}$ is the single point $f$, i.e., $x \in \operatorname{sm} S$. Thus, the norm in $E$ is strongly differentiable at $x$ and therefore $f \in \operatorname{str} S^{*}$, which was to be shown.

If $E$ is two-dimensional it is easy to verify that ext $U^{*}$ is dense in $S^{*}$ if and only if ext $U^{*}=S^{*}$, i.e., if and only if $E^{*}$ is strictly convex. This property is equivalent (see e.g. [6]) to the property that $E$ itself is $s m o o t h$, i.e., that $\operatorname{sm} S=S$. Thus we have the following corollary to Theorem 3.5.

Corollary 3.6. A two-dimensional normed linear space $E$ has property (g) if and only if $E$ is smooth.

5. An example. In this section we exhibit a separable Banach space $E$ which is isomorphic to $l_{1}$ (the space of absolutely summable sequences) and which has the following properties:

(i) $E$ is smooth and $E^{*}$ is strictly convex.

(ii) $E$ is subreflexive. ${ }^{1}$

(iii) The set $\operatorname{str} S^{*}$ is empty, hence the norm in $E$ is not strongly differentiable at any point of $S$.

(iv) The set $\exp U^{*}$ is dense in $S^{*}$.

(v) $E$ does not have property (g).

Now, smoothness of $E$ is equivalent to weak differentiability of the norm at each $x \neq \phi$ in $E$ (see, e.g. [1]), hence properties (i) and (iii) give a negative answer to the question, noted by Mazur in [5], as to whether weak differentiability always implies strong differentiability of the norm.

We will obtain the space $E$ by using a theorem of Day's [2, Theorem 5] to renorm $l_{1}$. We first define a new norm for $m=l_{1}^{*}$ (the space of bounded sequences $\left.y=\left\{y_{i}\right\}\right)$ by $\|y\|=\sup \left|y_{i}\right|+\left(\sum y_{i}^{2} / 2^{i}\right)^{1 / 2}$. If $x=\left\{x_{i}\right\}$ is in $l_{1}$, let $\|x\|=\sup \left\{\sum x_{i} y_{i}: y=\left\{y_{i}\right\} \in m\right.$ and $\left.\left\|y_{i}\right\|=1\right\}$. [To see how Day's theorem is applied to yield these norms, let the space $B_{0}$ of his theorem be $l_{2}$, let $B$ be $l_{1}$ and let $T$ be the mapping from $l_{2}$ into $l_{1}$ defined by $T\left(\left\{x_{i}\right\}\right)=\left\{x_{i} / 2^{i / 2}\right\}$.] Then, letting $E$ be $l_{1}$ so renormed, we can conclude from Day's theorem that $E$ is isomorphic with $l_{1}$, that $E^{*}$ is the space of bounded sequences with the norm described above, that $E$ is smooth and that $E^{*}$ is strictly convex. Thus, $E$ has property (i) above.

To prove that $\operatorname{str} S^{*}$ is empty, we first prove the following simple lemma:

If the norm in a linear space $E$ is strongly differentiable at the point $x \neq \phi$, if $f \in S^{*}$ is such that $f(x)=\|x\|$, if $\left\{f_{n}\right\} \subset E^{*}$ is such that 
$f_{n}(x) \rightarrow f(x)$ and $\left\|f_{n}\right\| \rightarrow\|f\|=1$, then $\left\|f_{n}-f\right\| \rightarrow 0$. To see this, note first that we can assume $\left\|f_{n}\right\|=1$ for all but a finite number of $n$, since the lemma is true for the sequence $\left\{f_{n}\right\}$ if and only if it is true for the sequence $\left\{f_{n} /\left\|f_{n}\right\|:\left\|f_{n}\right\| \neq 0\right\}$. Now if $\left\|f_{n}-f\right\| \rightarrow 0$ we can choose (taking a subsequence if necessary) $\epsilon>0$ and $y_{n} \in S$ such that $\left(f_{n}-f\right)\left(y_{n}\right) \geqq 2 \epsilon$. Now, let $x_{n}=\epsilon^{-1}\left[\|x\|-f_{n}(x)\right] y_{n}$ and use the fact that $\left\|x+x_{n}\right\| \geqq f_{n}(x)$ $+f_{n}\left(x_{n}\right)$ to show that $\left\|x_{n}\right\|-1\left[\left\|x+x_{n}\right\|-\|x\|-f\left(x_{n}\right)\right] \geqq \epsilon$, contradicting the assumption of strong differentiability at $x$ and the fact that $\left\|x_{n}\right\| \rightarrow 0$.

Thus, in order to show that no point $x \neq \phi$ in $E$ is a point of strong differentiability, it suffices to show that for each $x \neq \phi$ in $E$ and any $y \in S^{*}$ such that $(x, y)=\|x\|$ there exists a sequence $\left\{y^{k}\right\} \subset E^{*}$ such that $\left(x, y^{k}\right) \rightarrow\|x\|,\left\|y^{k}\right\| \rightarrow\|y\|$, but $\left\|y^{k}-y\right\| \rightarrow 0$. (The latter is equivalent, of course, to $\lim _{k \rightarrow \infty} \sup _{i}\left|y_{i}^{k}-y_{i}\right| \neq 0$.) Assuming we have such $x$ and $y$, we consider two cases. First, suppose that $y_{i} \rightarrow 0$. Pick an integer $n$ such that $\left|y_{i}\right|<1 / 4$ if $i>n$ and define $y^{k} \in E^{*}(k>n)$ by $y_{i}^{k}=y_{i}$ if $i \neq k, y_{k}^{k}=3 / 8$. Then $\left(x, y^{k}\right)=(x, y)-x_{k}\left(y_{k}-3 / 8\right) \rightarrow(x, y)$ (since $\left.x_{k} \rightarrow 0\right)$. Furthermore, since $\|y\|=1=\sup \left|y_{i}\right|+\left(\sum y_{i}^{2} / 2^{i}\right)^{1 / 2}$ $\leqq 2 \sup \left|y_{i}\right|$, we have $\sup \left|y_{i}\right| \geqq 1 / 2$. Hence

$$
\sup \left|y_{i}^{\mathbf{k}}\right|=\max \left(3 / 8, \sup _{i \neq \mathbf{k}}\left|y_{i}\right|\right)=\sup \left|y_{i}\right| \text {. }
$$

Thus, $\left\|y^{k}\right\|=\sup \left|y_{i}\right|+\left[\sum y_{i}^{2} / 2^{i}-\left(y_{k}^{2}-9 / 16\right) / 2^{k}\right]^{1 / 2} \rightarrow\|y\|$. Finally, $\left\|y^{k}-y\right\| \geqq \sup _{i}\left|y_{i}^{k}-y_{i}\right|=\left|3 / 8-y_{i}\right|>1 / 8$. Turning now to the other case, suppose there exists $\epsilon>0$ and a subsequence $\left\{y_{i_{k}}\right\}$ of $\left\{y_{i}\right\}$ such that $\left|y_{i_{k}}\right| \geqq 2 \epsilon, k=1,2,3, \cdots$. Define $y^{k} \in E^{*}$ by $y_{i}^{k}=y_{i}$ if $i \neq i_{k}, y_{i_{k}}^{k}=\epsilon$. Then $\left(x, y^{k}\right) \rightarrow(x, y)$ as before, and sup $\left|y_{i}-y_{i}^{k}\right|$ $=\left|y_{i_{k}}-\epsilon\right| \geqq \epsilon$. Furthermore, $\left\|y^{k}\right\| \rightarrow\|y\|$, which completes the proof of (iii).

To prove (iv), use the fact from (i) that $\operatorname{sm} S=S$, so $P(E) \cap S^{*}$ $C \exp U^{*}$, and apply (ii). To prove (v), we can use Theorem 4.3(iii) and the fact that $E$ (isomorphic to $l_{1}$ ) is separable, while $E^{*}$ (isomorphic to $m$ ) is not.

Parts (ii), (iv) and (v) of the above example show that the necessary conditions (subreflexivity and density of ext $U^{*}$ in $S^{*}$ ) given by Theorem 4.3 (i) are not sufficient. We know of no example, however, showing that the sufficient condition ( $\operatorname{str} S^{*}$ dense in $S^{*}$ ) of Theorem 2.2 is not necessary. While we believe such an example exists, it seems difficult to obtain.

\section{BIBLIOGRAPHY}

1. M. M. Day, Normed linear spaces, Berlin, Springer-Verlag, 1958.

2. - Strict convexity and smoothness of normed spaces, Trans. Amer. Math Soc. vol. 78 (1955) pp. 516-528. 
3. V. L. Klee, Extremal structure of convex sets. II, Math. Z. vol. 69 (1958) pp. 90104.

4. S. Mazur, Über konvexe Mengen in linearen normierten Räumen, Studia Math. vol. 4 (1933) pp. 70-84.

5. - Über schwach Konvergenz in den Räumen $\left(L^{p}\right)$, Studia Math. vol. 4 (1933) pp. 128-133.

6. R. R. Phelps, Subreflexive normed linear spaces, Arch. Math. vol. 8 (1957) pp. $444-450$.

7. - Some subreflexive Banach spaces, Arch. Math. vol. 10 (1959) pp. 162169.

INSTITUTE FOR ADVANCED STUDY

\section{ISOMETRIES OF GROUP ALGEBRAS ${ }^{1}$}

\section{PAUL CIVIN}

Let $G$ be a locally compact abelian group, $\hat{G}$ its character group, and $A$ the group algebra of $G$. Associated with any automorphism $\phi$ of $A[2]$ is a homeomorphism $\tau$ of $\hat{G}$ onto itself, with the property that, for $\alpha \in \hat{G}, f \in A$ and $\mathfrak{F}$ the Fourier transform, $\mathfrak{F}(\phi(f))(\tau \alpha)$ $=\mathfrak{F}(f)(\alpha)$. Results of Helson [2] and Wendel [4] state that if $e$ is the unit of $\hat{G}$, then

$$
\tau(e) \tau(x y)=\tau(x) \tau(y), \quad \text { for all } x, y \in \hat{G},
$$

if and only if $\phi$ is an isometry. The object of the present note is to give a further equivalent form of the statement that $\phi$ is an isometry.

Let $T_{\alpha}, \alpha \in \hat{G}$, be that operator on $A$ which for all $x \in G, f \in A$ satisfies $\left(T_{\alpha} f\right)(x)=f(x)(x, \alpha)$. We consider homomorphisms $\phi$ of $A$ onto $A$ such that to each $\alpha$ there is a $\rho(\alpha) \in \hat{G}$ such that

$$
\phi T_{\alpha}=T_{\rho(\alpha)} \phi, \quad \alpha \in \hat{G} .
$$

Our result is that such homomorphisms are isomorphisms and indeed isometries.

THEOREM 1. Let $\phi$ be a homomorphism of the group algebra $A$ of the locally compact abelian group $G$ onto itself. Suppose that $\phi$ satisfies (2); then $\phi$ is an isomorphism.

Let $K$ be the kernel of $\phi$. Since $\phi$ is automatically continuous [3], $K$ is a proper closed ideal of $A$. The Wiener Tauberian theorem thus yields a maximal regular ideal $M$ containing $K$. From condition (2) it follows that for $k \in K$ and $\beta \in \hat{G}, \phi T_{\beta}(k)=T_{\rho(\beta)} \phi(k)=0$, and there-

Received by the editors September 11, 1959.

1 This research was sponsored in part by the National Science Foundation under grant NSF-G5865. 